\begin{tabular}{|c|c|c|c|}
\hline Eiszeitalter u. Gegenwart & 30 & $\begin{array}{c}73-88 \\
10 \mathrm{Abb} ., 5 \mathrm{Tab} .\end{array}$ & Hannover 1980 \\
\hline
\end{tabular}

\title{
Paläoökologische Untersuchungen zum Krefeld-Interglazial am Niederrhein
}

\author{
BrigitTe Urban *) \\ Mit einem Beitrag von Jean-J. Puisségur \\ Herrn Prof. Dr. R. Schütrumpf zum 70. Geburtstag gewidmet \\ Pollen-diagram, flora, malakofauna, Saalian push-endmorain, Kempen-Krefeld-Interglacial, \\ middle terrace, Rhenisch Westphalian Basin (Lower Rhine Area)
}

TK 25: Nr. 4304, 4404, 4405, 4504, 4505, 4506

Kurzfassung: Aus dem Bereich der saalezeitlichen Stauchendmoränen im Großraum Krefeld am Niederrhein wurden vier Pollendiagramme aus den Krefelder-Schichten erarbeitet und als Krefeld-Interglazial bezeichnet.

Das Profil Römerhof II wurde zudem malakozoologisch untersucht. Die erfaßten vegetationsgeschichtlichen Abschnitte zeigen bei relativer Gleichförmigkeit eine Dominanz der Koniferengehölze, die mit Auewaldelementen (Pterocarya, Alnus, Vitis) das Vegetationsbild am Niederrhein ausgemacht haben.

Juglandaceae-Pollen ist ebenso wie der Pollen von Fagus in diesen Abschnitten des Interglazials regelmäßig, allerdings nur in Spuren vertreten. Massulae von Azolla filiculoides sind in allen Profilen zum Teil reichlich vorhanden.

Eine stratigraphische Einstufung der gestauchten (Krefelder-Schichten) und der ungestauchten (Kempener-Schichten) wird diskutiert.

\section{[Paleoecological Investigations of the Krefeld-Interglacial / Lower Rhine Area]}

A bstract: In the area of Krefeld (Lower Rhine Region) clay-horizons being intercalated inbetween Saalian push-endmoraines have been analysed for pollen. Four pollen-diagramms of those Krefelder-Schichten will be discussed in this paper.

The warm period found is termed Krefeld-Interglacial.

The profile of the locality Römerhof II has as well been analysed for malakozoological remains.

The type of vegetation prevailing during the probably second part of the interglacial is characterized by a predominance of coniferous trees and a riverrhine vegetation (Pterocarya, Alnus, Vitis). Pollen of Juglandaceae has been found as well as pollen of Fagus in this interglacial period repeatedly, but only with very few grains. All profiles are abundant in Massulae of the water fern Azolla filiculoides.

The stratigraphic position of the glacigene pushed deposits (Krefelder-Schichten) and the in situ located deposits (Kempener-Schichten) is discussed at the end of the paper.

\section{Einleitung}

In der weiteren Umgebung von Krefeld, also in dem Bereich, in welchem die Krefelder Mittelterrasse verbreitet ist, und den rheinwärts angrenzenden saale-zeitlichen Stauchendmoränen (Abb. 1) sind schluffig-tonige Altwassersedimente bekannt. Sie unterteilen als „Kempener-Schichten“ ungestörte Schotter, und als „Krefelder-Schichten“ sind sie in die Stauchendmoränen eingeschuppt. KEмPF (1966) hat beide Gruppen als „Kempen-Krefelder-Schichten " zusammengefaßt.

*) Anschriften der Verfasser: Dr. B. U r b a n, Institut für Bodenkunde der Universität Bonn, Nußallee 13, 5300 Bonn; Dr. J. J. P u is s é g u r, Hauteroche, F 21250 Les Laumes. 

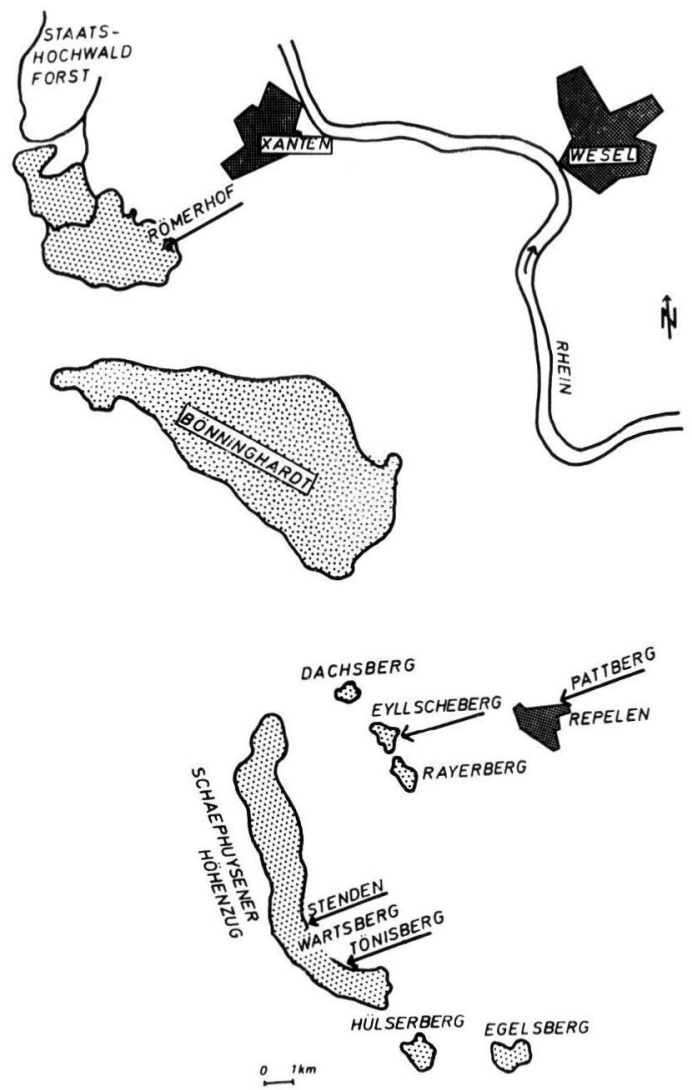

Abb. 1: Lage der Profile in den Stauchwällen und Inselbergen am Niederrhein.

Konventionellerweise werden diese feinkörnigen Ablagerungen dem Holstein-Interglazial zugeordnet, (zusammengefaßt bei KEMPF 1966). Aus der älteren Literatur (BERTSCH \& Steeger 1927; Bertsch, Steeger \& Steussloff 1931 und Steeger 1952) sind weitere ähnliche Bildungen als „Moerser Schichten“ bekannt geworden. Neben der faziellen Ausbildung wurden paläobotanische und malakozoologische Befunde zu deren Definition genutzt.

Für eine Trennung der Kempener-Schichten und der Krefelder-Schichten aufgrund stratigraphischer und faunistischer Befunde sprechen die Argumente von KAISER \& SCHÜTRUMPF (1960).

Im Anschluß an die paläobotanische Bearbeitung des Altestpleistozäns der Niederrheinischen Bucht (URBAN 1978 a, b, c) wurden einige dieser mittelpleistozänen Ablagerungen pollenanalytisch untersucht.

Es handelt sich dabei um Vorkommen im Bereich der innerhalb des Niederterrassenareals liegenden Einzelberge (Inselberge), dem „Eyll'sche-Berg“; dazu kommt im Bereich des Schaephysener Höhenzuges ein ebenfalls gestauchtes, nach der Lokalität Stenden bezeichnetes Vorkommen am Wartsberg. In der Stauchmoräne SW Xanten liegt die als Römerhof bezeichnete dritte Lokalität (Abb. 1). 
Die jüngsten geologisch-paläontologischen Untersuchungen zu derartigen Ablagerungen stammen aus dem Schacht Tönisberg (Abb.1) von KempF (1966), vom Südende des Schaephysener Höhenzuges.

Herr Prof. Dr. KempF hat freundlicherweise Material zur Einsichtnahme zum Vergleich zur Verfügung gestellt; dafür sei ihm an dieser Stelle gedankt.

Ferner liegen Ergebnisse von GrüNER (1975) und LANSER (1978) über den geologischen Aufbau im Bereich der Krefelder Terrasse vor; diese Untersuchungen werden weitergeführt.

\section{Die untersuchten Profile}

2.1. Entnahme, Aufbereitung und Auszählen der Pollenpräparate und Diagrammdarstellung

Sämtliche Proben wurden an Aufschlußwänden entnommen und zwar in lückenlosen Abständen in 10-20 cm langen Blöcken.

Die Probenaufbereitung erfolgte in der bewährten Weise (nach Frenzel 1964, modifiziert) mit einer K-Cd Jodid-Schweretrennung (ausführliche Beschreibung bei URBAN 1978 c). Auf diese Art und Weise konnte eine Anreicherung einer zum größten Teil ausreichenden Sporomorphenmenge der durchweg anorganischen, schluffig-tonigen Bildungen erreicht werden.

Beim Auszählen der Pollendiagramme wurde darauf geachtet, einen möglichst repräsentativen Querschnitt eines Deckgläschens zu erhalten, wenn genügend Material zur Verfügung stand. Bei geringer Pollendichte wurden ein bis mehrere Deckgläschen $(21$ x $26 \mathrm{~mm})$ ausgezählt.

Bei der Darstellung der Pollendiagramme wurden nur einige wesentliche Taxa als Schattenrißkurven abgebildet (Abb. 1, 2, 3, 4), selten auftretende Pollenformen können den Tabellen 1, 2, 3 und 4 entnommen werden. Als Basissumme diente aller Baumpollen einschließlich Corylus und aller Kräuterpollen. Pollen aquatischer Pflanzen, Farn- und Moossporen sowie Ericaceenpollen ist aus der Basissumme ausgeschlossen. Bei den Detaildiagrammen (Abb. 6-9) wurde Alnus, als Lokalfaktor, aus der Basissumme herausgenommen.

2.2. Die Polle ndiagra m me (Zonierung siehe Detaildiagramme, Abb. 6-9)

Römerhof I TK 50 L 4304 Wesel (R 25/284000 H 57/201600)

Das Pollendiagramm weist eine Drei- (bzw. Vier-)Teilung auf:

Lokalgliederung: (Abb. 2 und 6)

Abschnitt 1: $\quad 370-205 \mathrm{~cm}$

An der Basis wird das Diagramm von Corylus bestimmt, bei geringer Beteiligung von Pinus, Abies und den Eichenmischwaldelementen. Aquatische Pflanzen spielen auch eine Rolle und von besonderer Bedeutung ist das erste Auftreten von Azolla filiculoides (Massulae).

Abschnitt 2: $\quad 205-115 \mathrm{~cm}$

Es folgt ein Abschnitt mit hohem Pinus-Anteil, mit Abies und wenig Corylus. In der Probe R 17 (Abb. 2) tritt neben Pterocarya auch Vitis zum erstenmal in diesem Profil auf. Die Eichenmischwaldanteile sind gering. 


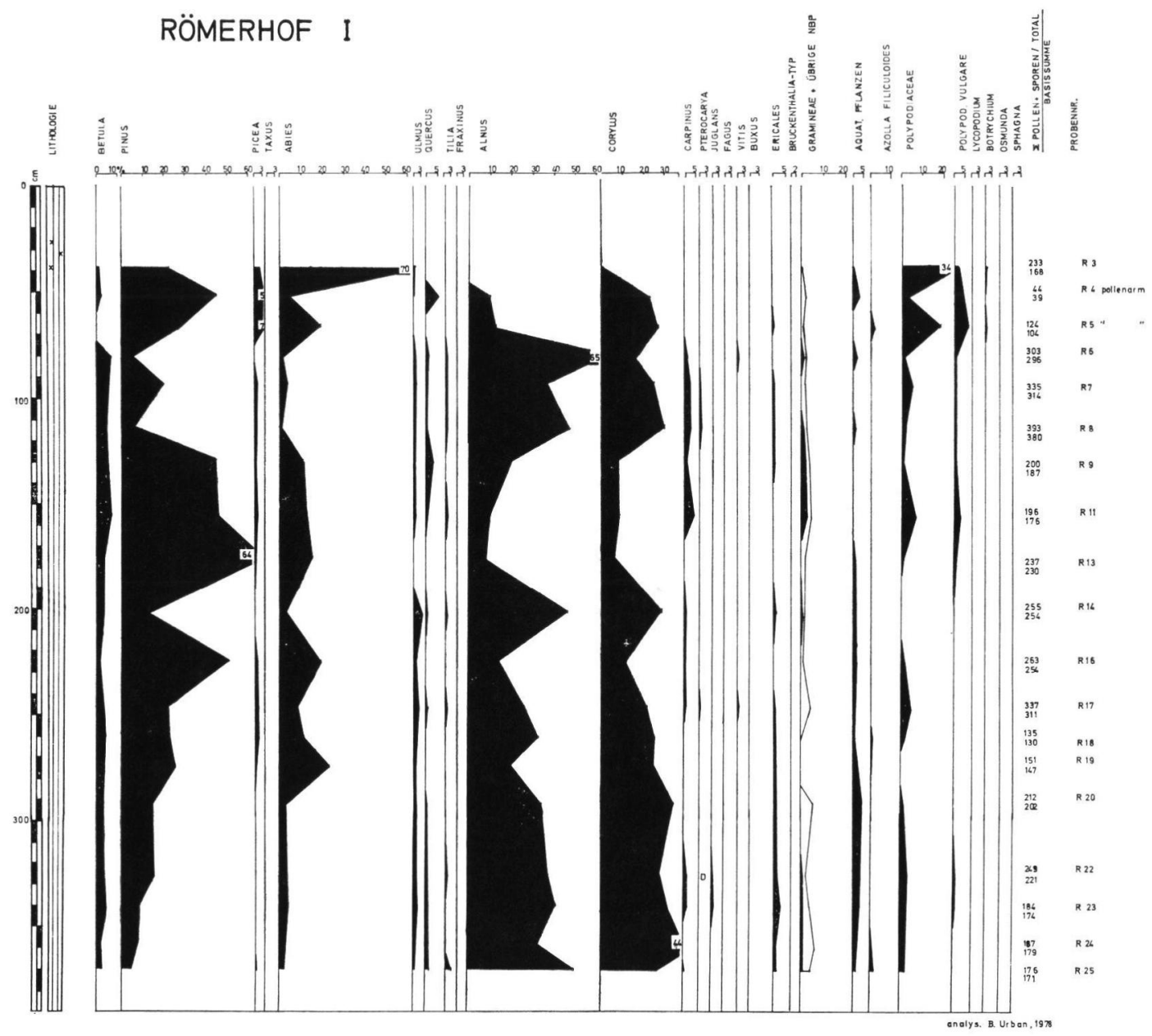

Abb. 2: Pollendiagramm, Römerhof I.

Abschnitt 3: $\quad 115-40 \mathrm{~cm}$

Es zeichnet sich eine erneute Haselphase ab, die allerdings einen scheinbar kürzeren Zeitraum einnimmt als die des Abschnittes 1, mit Pinus- und Abies-Beteiligung, dazu kommen in geringem Maße Eichenmischwald- und Hainbuchen-Anteile.

Das Pollendiagramm endet mit einer erneuten Zunahme von Pinus zusammen mit Picea, wobei Abies einen Gipfel von $70 \%$ erreicht (Probe R 3, Abb. 2). Alnus und Corylus gehen in den obersten Profilabschnitten auf annähernd $0 \%$ zurück (Abb. 2 und 6).

Römerhof II

Lokalgliederung: (Abb. 3 und 7) 


\section{RÖMERHOF II}

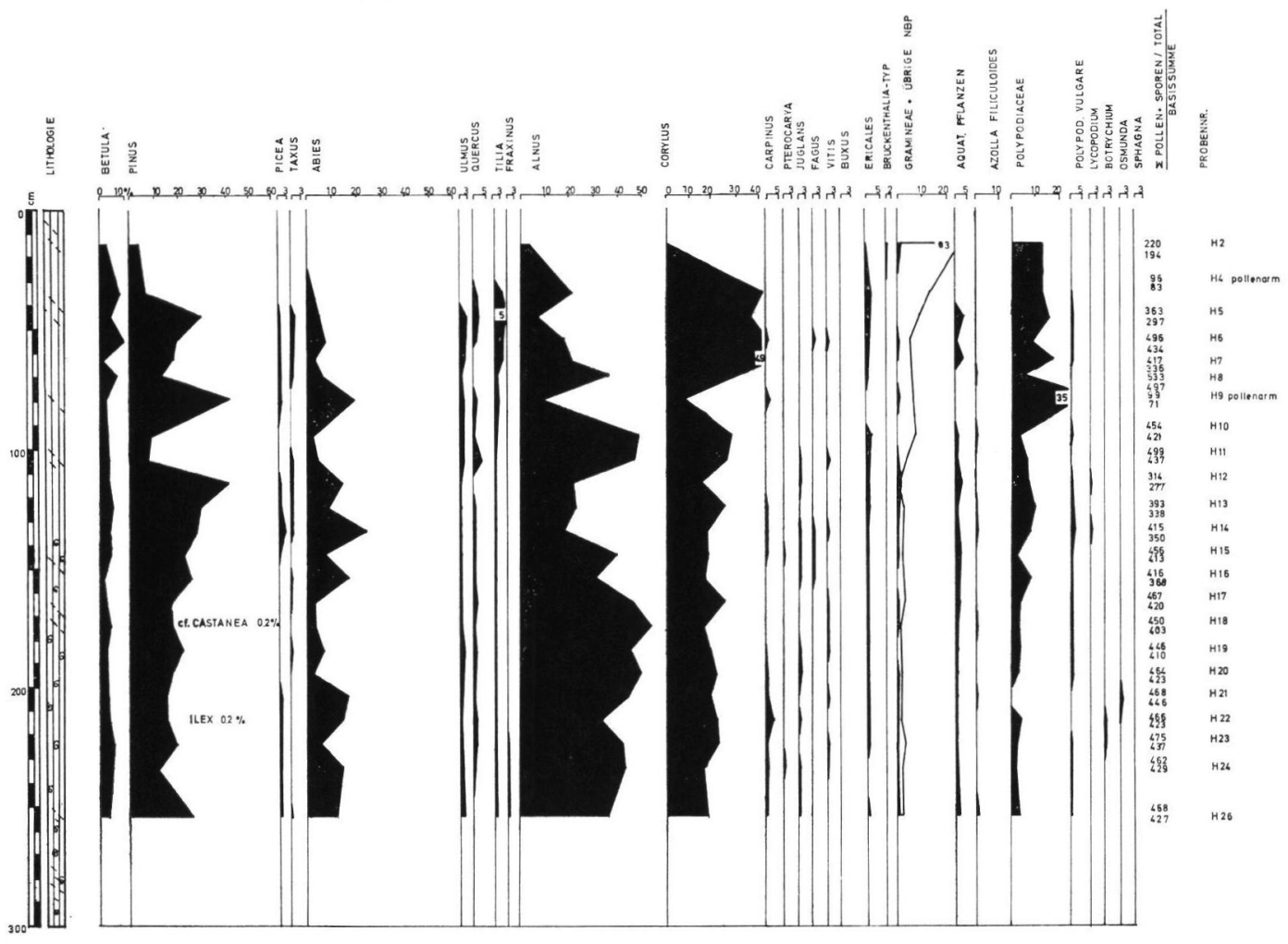

Abb. 3: Pollendiagramm, Römerhof II.

Abschnitt 1: $\quad 255-90 \mathrm{~cm}$

An der Basis des Pollendiagramms zeichnet sich eine recht gleichförmige Phase ab: über insgesamt fast die Hälfte des Diagramms dominieren Hasel und Kiefer, Abies und der Eichenmischwald sind ebenfalls beteiligt, in geringem Maße die Hainbuche. Es treten mit geringen Werten Pterocarya, Juglans und Vitis auf. Auch Massulae von Azolla filiculoides sind gefunden worden.

Abschnitt 2: $\quad 90-15 \mathrm{~cm}$

Es kommt im weiteren Diagrammverlauf zu einer Hasel-Dominanz (Abb. 7), mit Kiefer und wenig Tanne, aber höheren EMW- und Taxus-Anteilen. Juglans-Pollen ist wiederholt in dieser Zone gefunden worden, ebenso wie Fagus-Pollen (Abb. 3). Von diesem wird angenommen, daß er auf primärer Lagerstätte liegt, da sich durch die übrige Pollenvergesellschaftung kein Hinweis auf Umlagerung erkennen läßt.

Nach einer erneuten Erlendominanz nehmen die Corylus-Werte stark zu (Abb. 3). In der Probe $\mathrm{H} 6$ tritt erneut Fagus-Pollen auf.

Im jüngsten Teil dieses Abschnittes findet eine starke Zunahme des NBP statt; insbesondere ergeben sich diese hohen Werte durch Cruciferenpollen. Zudem tritt in diesen oberen Proben Pollen vom Bruckenthalia-Typ auf (Abb. 3). Dieser Pollentyp ist von 
URBAN (1978 b) für den ältestpleistozänen Interglazialtyp, „van Eyck-Interglazial“ im Raum Brüggen (nordwestliche Niederrheinische Bucht) beschrieben worden und findet sich auch in zeitgleichen Diagrammen von Menke (1975) in Schleswig-Holstein. Er ist in allen wesentlichen Merkmalen mit dem von BEHRE (1974) für jungpleistozäne Ablagerungen beschriebenen Bruckenthalia-Pollen ident; es handelt sich jedoch um eine wesentlich kleinere Form. Der von Turner (1970) vorgestellte Pollen Erica terminalis in den Ablagerungen von Hoxne kommt dieser kleinen Bruckenthalia-Form sehr nahe.

Stenden TK 50 L 4504 Moers (R 25/362000 H 57/0416000)

Lokalgliederung: (Abb. 8 und 4).

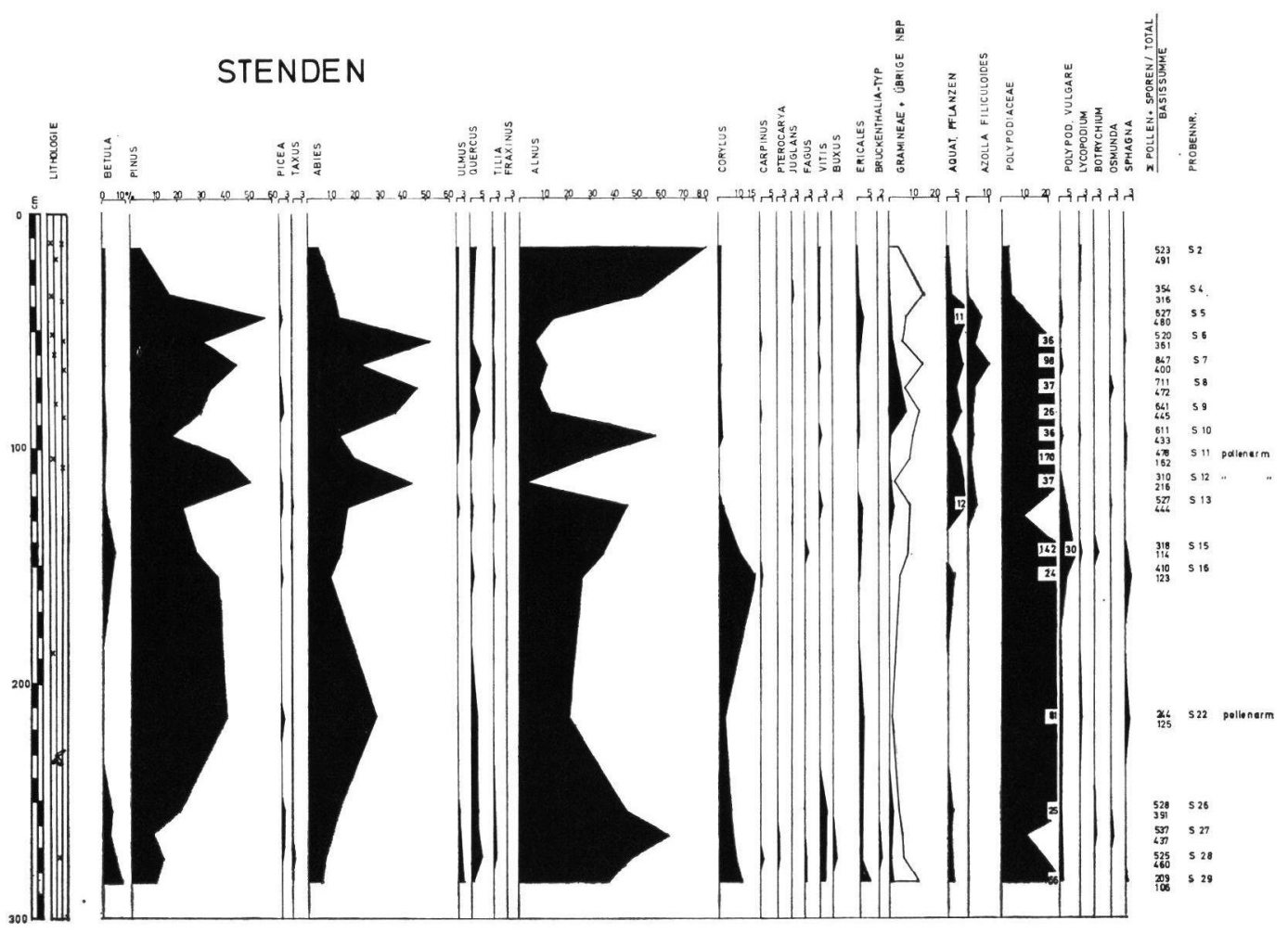

Abb. 4: Pollendiagramm, Stenden.

\section{Abschnitt 1: $\quad 285-120 \mathrm{~cm}$}

Der basale Abschnitt wird dominiert von Abies und Pinus, wobei auch Corylus eine wenn auch geringe Rolle spielt. Im tiefsten Teil des Diagramms tritt neben PterocaryaPollen auch wieder Fagus-Pollen auf, und bei leicht erhöhten Vitis-Werten konnte auch Buxus-Pollen gefunden werden (Abb. 4).

$\mathrm{Ab}$ der Probe S 13 tritt mit erhöhten Werten und in geschlossener Kurve Azolla filiculoides (Massulae) auf. 
Abschnitt 2: $\quad 120-15 \mathrm{~cm}$

In den oberen Proben erfolgt eine starke Eichenmischwaldausbreitung bei hohen Pinus- und Abies-Anteilen, Corylus sinkt weiter ab.

Eyll'sche Berg TK 50 L 4504 Moers (R 25/321100 H 56/981400) (Abb. 5 und 9)

Das Probenmaterial war von der Pollenführung her weitgehend unbefriedigend. Nur im oberen Profil-Teil war die Pollenführung zufriedenstellend und prozentual auswertbar.

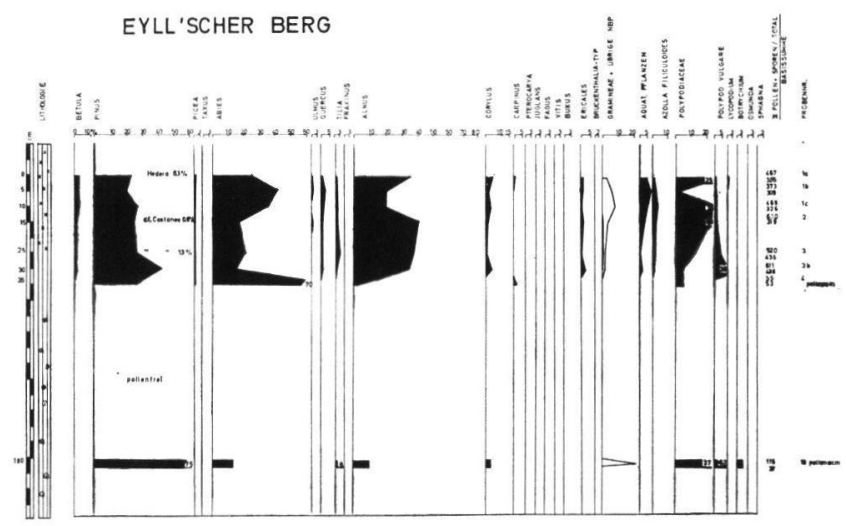

Abb. 5: Pollendiagramm, Eyllsche Berg.

Der obere Diagrammabschnitt kann als eine Einheit aufgefaßt werden. Unter den Gehölzen sind Pinus und Abies gleichermaßen stark beteiligt. Corylus liegt ebenso wie die Eichenmischwaldelemente um 5\% (Abb.9). Über den gesamten Abschnitt hinweg läßt sich eine geschlossene Kurve von Azolla filiculoides (Massulae) beobachten (Abb. 5).

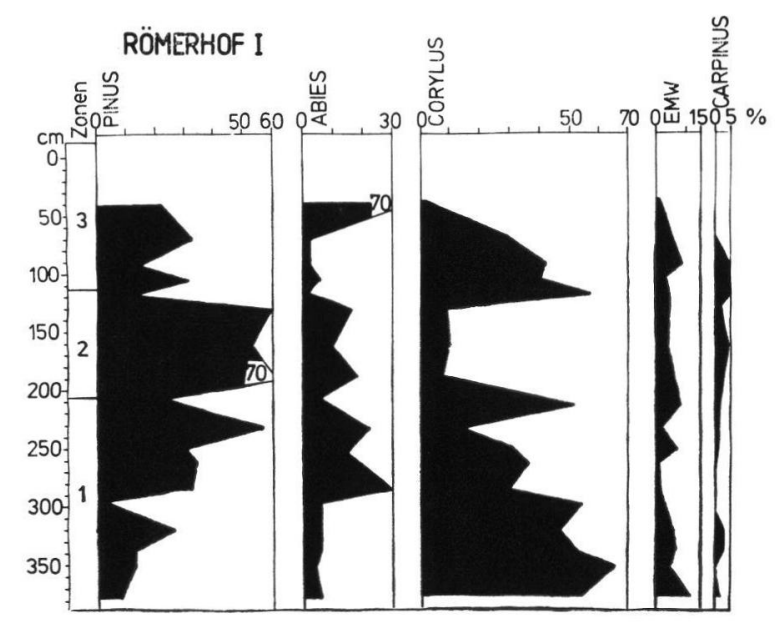

Abb. 6: Detaildiagramm, Römerhof I. 

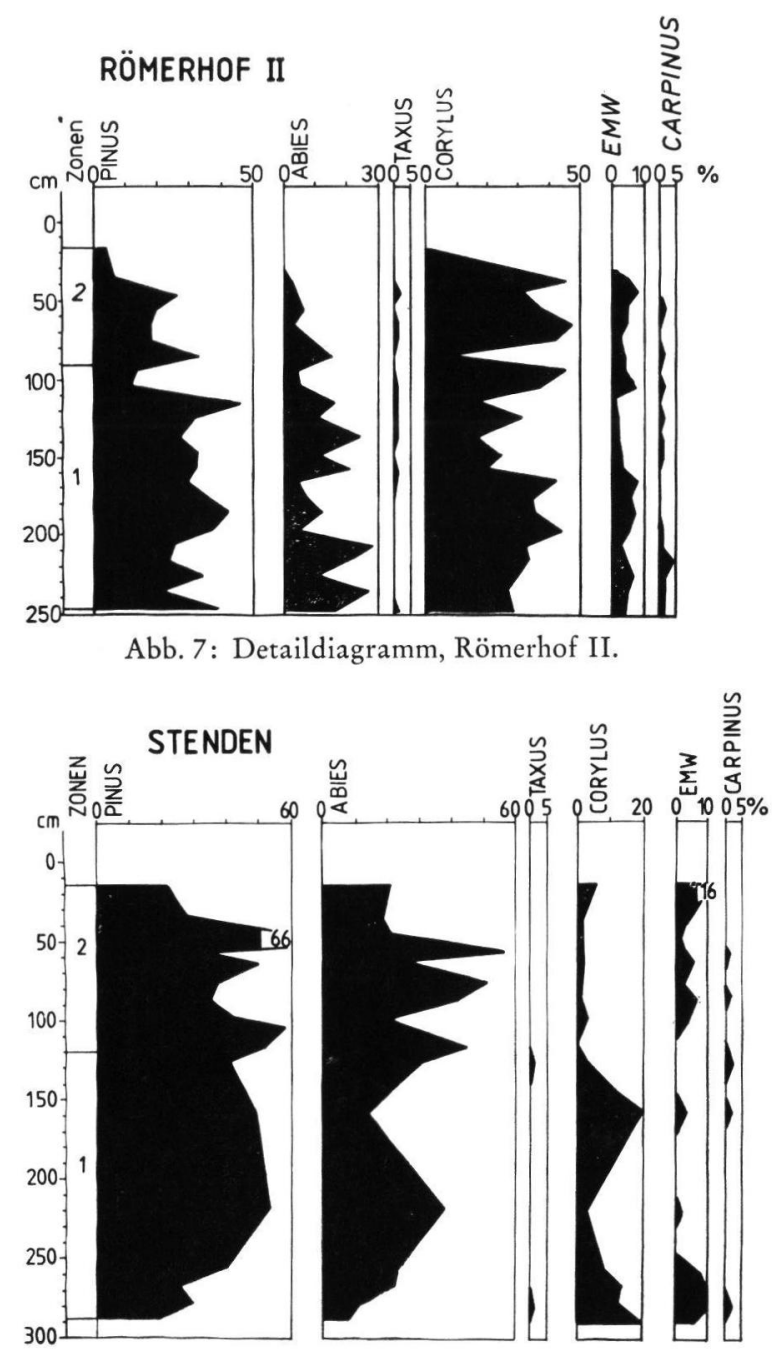

Abb. 8: Detaildiagramm, Stenden.

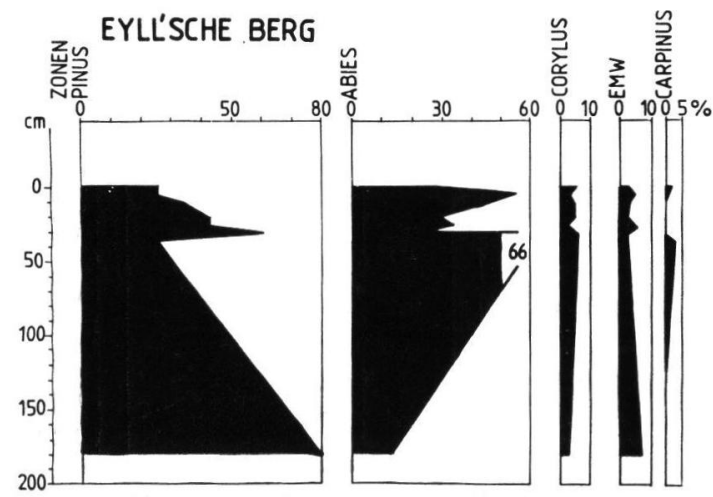

Abb. 9: Detaildiagramm, Eyllsche Berg. 


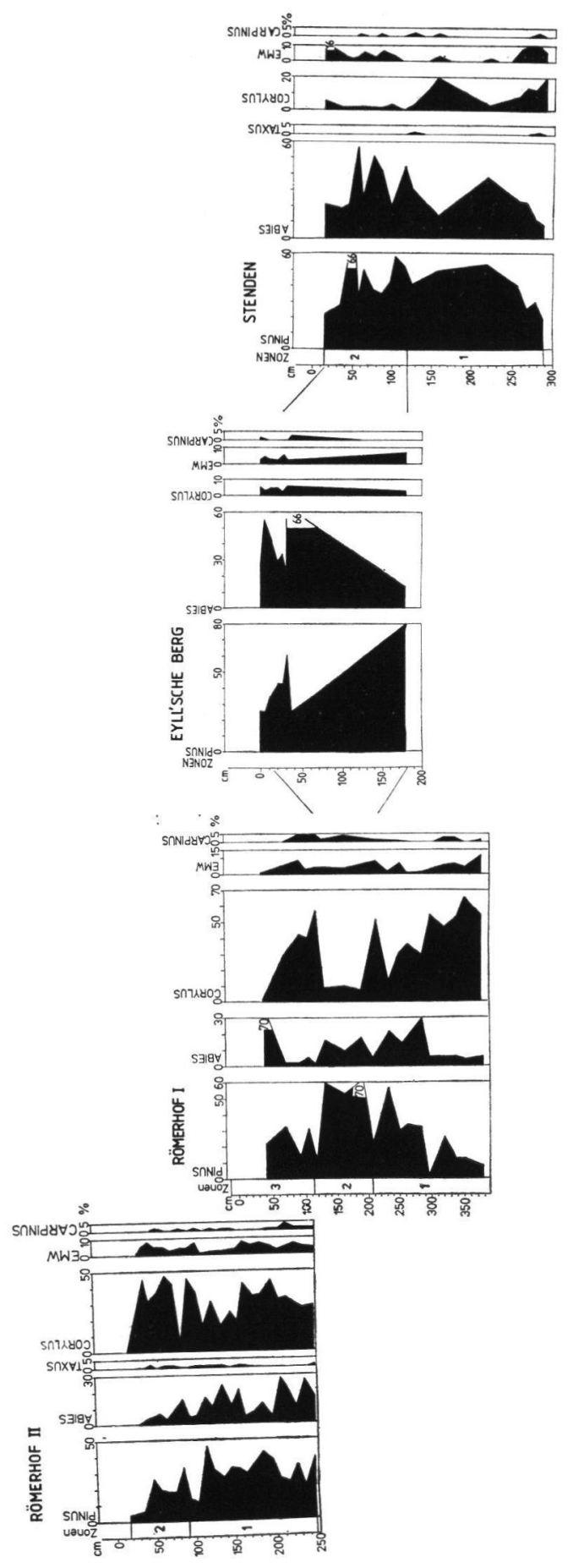

Abb. 10: Parallelisierung der Profile über Pollenzonen. 


\section{Diskussion der Pollendiagramme der Krefelder-Schichten und Vergleich mit zeitgleichen Vorkommen}

Die sich pollenfloristisch entsprechenden Abschnitte sind miteinander korreliert worden, wobei eine übergeordnete Zonierung eingeführt wurde.

Aus der Abb. 10 geht hervor, daß sich die Diagramme aufgrund einiger charakteristischer Phasen und Tendenzen in Verbindung mit selten auftretenden Pollenfunden gut gliedern und miteinander parallelisieren lassen.

Die älteste erfaßte Zone 1 (im Diagramm Römerhof I) zeichnet sich durch die hohe Hasel-, Kiefer- und Tannenbeteiligung aus. In diesem basalen Teil wurden bereits Massulae von Azolla filiculoides gefunden, ebenso wie Pollen von Vitis, Buxus, Juglans, Pterocarya und Fagus. Die zuletzt genannten Formen treten allerdings nur in Spuren auf (zumeist unter $1 \%$ ).

Zone 1 von Stenden zeigt im Gegensatz zu Zone 1 von Römerhof I einen Wechsel im Pollenspektrum zugunsten von Pinus und Abies. Auch in dieser Phase ist Pollen von Pterocarya, Juglans und Fagus gefunden worden. Diese Zone könnte zwischen Zone 1 und 2 des Profiles Römerhof I eingestuft werden.

Zone 2 von Römerhof I ist als eine Parallele zu Zone 2 von Stenden und dem Profil des Eyll'schen Berges aufzufassen, wobei bei Pinus- und Abies-Dominanz die Beteiligung von Corylus gering ist. Die Eichenmischwaldelemente sind jedoch zusammen mit Carpinus zum Teil stärker vertreten.

Die folgende Zone 3 von Römerhof I könnte mit der Zone 1 des Pollendiagramms Römerhof II parallelisiert werden. Die Kiefern- und Hasel-Anteile sind ungefähr gleich hoch. Die Tanne und die Eichenmischwaldelemente, in geringerem Maße auch die Hainbuche, spielen eine Rolle.

Die Zone 2 im Profil Römerhof II geht demnach über alle anderen Diagramme hinaus; Corylus herrscht vor, Pinus und Abies sind mit geringeren Werten als zuvor vertreten bei relativ guter Beteiligung des Eichenmischwaldes und Taxus.

Insgesamt betrachtet muß während der diskutierten Zonen mit einem recht gleichförmigen Vegetationstyp gerechnet werden. Dominant im Waldbild waren vor allem Nadelgehölze, wobei ein ausgedehntes Auewaldsystem angenommen werden kann (KEMPF 1966), in dem die Erlen, aber auch die Hasel mit Pterocarya, Vitis und dem Wasserfarn Azolla filiculoides am Niederrhein vertreten waren. Für diese Warmzeit mit ihrem charakteristischen pollenfloristischen Bild wird hier der Begriff „Krefeld-Interglazial“ eingeführt. Da nur ein Ausschnitt aus einem vermutlich wesentlich umfangreicheren Interglazial (ZAGwIJN 1973; Müller 1974) erfaßt werden konnte, ist anhand der ermittelten Abschnitte ein Klimaverlauf nur andeutungsweise zu rekonstruieren. Immerhin kann aber, abgesehen von der zeitlich-stratigraphischen Einstufung, über das Klima während der Ablagerungszeit folgendes gesagt werden: es herrschten warm-humide Klimabedingungen, wobei der hohe Koniferenanteil nicht zwingend auf eine sich bereits anzeigende Klimaverschlechterung hindeuten muß. Der hohe Koniferenanteil kann auch auf die sich verändernden edaphischen Bedingungen im Verlauf einer Warmzeit zurückzuführen sein. Schließlich spielen Standortfragen hinein, die im Niederungsgebiet am Niederrhein einen raschen räumlichen Wechsel zwischen Auewald und trockenen Standorten der anspruchslosen Gehölze zur Folge haben.

Bei einem ersten Vergleich mit dem wohl bisher am vollständigsten erfaßten holsteinzeitlichen Vorkommen von Munster-Breloh (MüLLER 1974) zeigt sich darüberhinaus auch, daß die hier am Niederrhein erfaßten interglazialen Bildungen bereits nahezu an das Ende 
der Holstein-Warmzeit gestellt werden könnten. ZAGWIJN (1963) beschreibt das Auftreten vom Pterocarya-Pollen neben Müller aus dem Abschnitt Ho 3 b seiner Gliederung des Holstein-Interglazials und damit aus einer jüngeren Phase dieser Warmzeit.

Die Sedimentation der schluffig-tonigen Altwasserablagerung hat somit, und zwar für alle der hier diskutierten Vorkommen, am Niederrhein erst gegen Ausgang des Holstein-Interglazials eingesetzt. Eine beginnende Sedimentation erst zum Ende der Warmzeiten hin konnte allerdings bisher in der Niederrheinischen Bucht zumeist beobachtet werden. So setzen ältestpleistozäne warmzeitliche Tonablagerungen im Raum Brüggen im Klimaoptimum des Interglazials ein (van Eyck-Interglazial, URBAN 1978 b). Auch für ältestpleistozäne Vorkommen auf der Ville konnten bisher keine Übergänge mit Einwanderungs- und Ausbreitungsphasen der Gehölze in entsprechenden Altwassersedimenten ermittelt werden. So lassen sich die interglazialen Bildungen (z. B. Frechen I Interglazial, Tiglium A) zwar zum Teil in unterschiedliche vegetationsgeschichtliche Abschnitte untergliedern, setzen jedoch meist ebenfalls erst im thermischen Optimum oder später ein und lassen häufig eine schnelle Sedimentation toniger Fazies erkennen. Diese hält zum Teil bis in die Frühglazialphasen an, falls nicht in Zusammenhang mit hangenden Flußsedimenten gekappt.

Das von KEMPF (1966) beschriebene Pollendiagramm aus den ungestörten KrefelderSchichten von Schacht Tönisberg gehört offenbar ebenfalls an das Ende des Holstein-Interglazials. Es dürfte jedoch im Vergleich mit den eigenen Untersuchungen einem etwas älteren Abschnitt zuzuordnen sein, den ZAGWIJN (1973) als Ho 2 / Ho 3a bezeichnet. Charakteristisch sind im Schacht Tönisberg die noch recht hohen Quercus-Anteile zusammen mit Pinus und Abies. Eine weitere genauere Parallelisierung mit diesem Diagramm ist jedoch nicht möglich; anhand eigener Nachuntersuchungen konnten die hohen Pterocarya- und Castanea-Werte in diesem Profil nicht bestätigt werden. Auch sind bei KempF keine Hinweise auf das Vorkommen von Fagus-, Vitis-, Buxus- und Taxus-Pollen anhand des Pollendiagramms zu finden. Vitis sylvestris beschreibt er ebenso wie Azolla filiculoides (interglazialica) makroskopisch. (Möglicherweise zeitgleich können Spektren aus dem KärlicherInterglazial sein, worauf die hohen Quercus- und Pinus-Anteile hindeuten (URBAN, 1978d).

VON DER BRELIE et al. (1959) beschreiben aus der Rinnenfüllung im Flöz Frimmersdorf eine Reihe makroskopisch und mikroskopisch erfaßter Pflanzenreste, die ein holsteinzeitliches Alter dieser Ablagerungen nahelegen könnten. Auch in diesen Bildungen wurden Reste von Taxus baccata, Pterocarya cf. fraxinifolia und Buxus sempervirens gefunden, die übrigen pollenanalytischen Befunde deuten auf kühle Bedingungen während des Ablagerungszeitraumes hin. Allein aufgrund der Lagerungsverhältnisse wurden diese Vorkommen seinerzeit in das Mindel/Riß-Interglazial gestellt. Dieses Frimmersdorf-Interglazial liegt in der Mittelterrasse II (BRUNNACKER et. al. 1978) und ist damit deutlich älter als das „Krefeld-Interglazial“. Auf die abgeschlossenen palynologischen Untersuchungen (UrbAN 1979) zu einem Ausschnitt aus dem Frimmersdorf-Interglazial wird an anderer Stelle eingegangen.

Die „Vogelheimer Flora“ (KRÄUSEL 1937) bei Essen weist, abgesehen von vielen, später von anderen Autoren beschriebenen typisch-holsteinzeitlichen Taxa, ebenfalls auf ein älteres Interglazial, da bisher Tsuga-Pollen noch nicht in eindeutig holsteinzeitlichen Ablagerungen gefunden wurde.

\section{Malakozoologische Befunde (PUISSÉGUR)}

Die Malakofauna des Profils Römerhof II ist ausgesprochen arm und das Material zum Teil schlecht erhalten. Alle Formen sind aquatisch, was auch mit dem Vorkommen von Fischzähnen in Übereinstimmung zu bringen ist. Valvata piscinalis ist häufig gefunden worden (vgl. Tab. 1) und die Gehäuse sind gut erhalten. Von Bithynia tentaculata 
sind, obwohl deren Gehäuse resistent ist, nur Deckel vorhanden. Die gefundenen Reste von Viviparus sind zu fragmentarisch, um eine Artbestimmung vornehmen zu können.

Tab. 1: Molluskenfauna des Profils Römerhof II

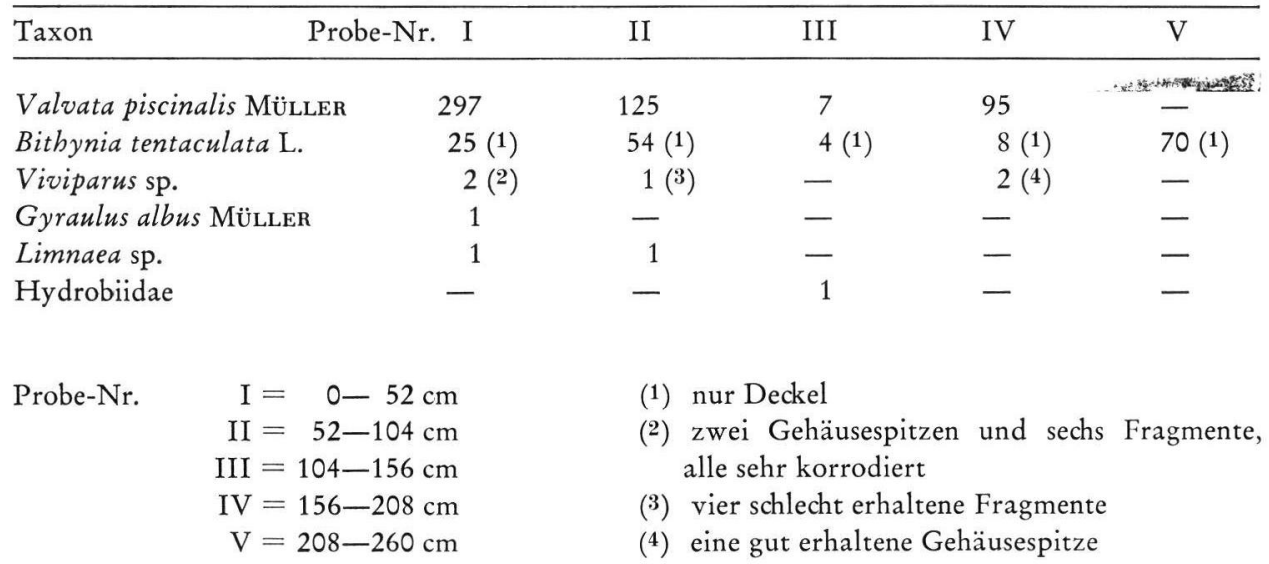

Ebenfalls bleibt offen, um welche Spezies es sich bei dem Hydrobiidae-Exemplar handelt. Es ist dabei nicht auszuschließen, daß es sich bei den aufgefundenen Resten von Bithynia tentaculata und Viviparus um Bestandteile aus einer älteren, hier eventuell sekundär eingeschwemmten Ablagerung handelt.

\section{Stratigraphische Bemerkungen}

Es ist naheliegend, die paläobotanisch und zum Teil malakozoologisch bearbeiteten Vorkommen konventionellerweise in das Holstein-Interglazial im Sinne eines vorletzten Interglazials einzustufen.

Daneben ist jedoch für dieses „Krefeld-Interglazial“ eine weitere, nämlich eine um eine Warmzeit ältere Einstufung in Ansätzen diskutabel, im Sinne einer drittletzten Warmzeit (ebenfalls Holstein-Interglazial). Für eine solche Möglichkeit können sprechen:

a) paläobotanische Befunde

Die rein paläobotanischen Befunde für sich allein gestellt lassen eine Parallelisierung mit Profilen des klassischen Eems nicht zu. Auch ist eine eindeutige Einstufung in das Holstein-Interglazial nicht gesichert, wenngleich sich gewisse Beziehungen zu Ausschnitten der Holstein-Gliederungen von ZAGwiJn (1974) und MüLLER (1974) herstellen lassen. Das Pollendiagramm (KEMPF 1966) von Schacht Tönisberg zeigt jedoch kaum Parallelen zu denen des Krefeld-Interglazials. Immerhin stehen damit folgende Kriterien zur Diskussion: dem Interglazialtyp des „Krefeld-Interglazials“ fehlen die eventuellen frühen $\mathrm{Ab}$ schnitte, die für eine Charakterisierung der Florengeschichte Voraussetzung sind. Ferner ist das Vorkommen von insbesondere Juglandaceen (Pterocarya, Juglans), abgesehen von ältest-pleistozänen Ablagerungen, nicht allein auf das Holstein-Interglazial beschränkt (z. B. Frimmersdorf-Interglazial, vON DER BRELIE et al. 1959), und daher für die stratigraphische Aussage für sich allein gestellt nur bedingt brauchbar. 


\section{b) Mollusken}

In seiner zusammenfassenden Arbeit über die Mollusken des Holstein-Interglazials des Niederrheingebietes faßt KEMPF (1968) alle, auch von anderen Autoren bis dahin beschriebenen Formen der „Kempen-Krefelder" Schichten zusammen. Dabei wird auf das stratigraphisch interessante Vorkommen unter anderem von Viviparus diluvianus hingewiesen. Bis auf Limnaea und das Hydrobiidae-Exemplar sind die in Römerhof II gefundenen Mollusken auch schon bei KEMPF $(1966,1968)$ beschrieben. Sie bestätigen die interglazialen Ablagerungsbedingungen und deuten ökologisch betrachtet wechselnde Strömungsgeschwindigkeiten eines Flußlaufes an.

\section{c) Geologie}

Auch aus der Sicht des geologischen Aufbaus ist ferner die Möglichkeit einer älteren Einstufung nicht völlig ausgeschlossen (BRUNNACKER et al. 1978; BRUNNACKER 1978). So sind im Areal der östlich angrenzenden Niederterrasse einige Sandvorkommen im Liegenden der Niederterrasse bekannt, die Azolla filiculoides führen. Dieses Florenelement ist aber bisher nur aus präeemzeitlichen Vorkommen in NW-Europa bekannt. Wenngleich diese Ablagerungen mit Azolla filiculoides umlagert sein können, so fragt sich doch, ob es sich um über weitere Strecken verlagertes Material handelt, oder um nur kurzstreckig, lokal verlagerte Sedimente, oder ob diese Sedimente nicht wirklich in situ liegen.

\section{d) Ergebnis}

Anhand der dargestellten Argumente ergibt sich für eine stratigraphische Zuordnung der im allgemeinen gestauchten Krefelder-Schichten (Krefeld-Interglazial) folgendes: Die interglazialen Vorkommen in den Stauchwällen und Inselbergen (Krefeld-Interglazial) sind gleichalt und gehören in eine ältere Warmzeit (Holstein).

Die Kempener Schichten stehen im Raum Krefeld in engem räumlichen Verband mit den gestauchten Krefelder Schichten. Dies war einer der Gründe für KEMPF (1966), beide Ablagerungen zusammenzufassen (Kempen-Krefelder Schichten). Aber auch wenn man diese Parallelisierung akzeptiert, so ist doch zum einen die Stellung der Moerser Schichten als ebenfalls gleichaltrig (nach KEMPF) nicht präzise gesichert, noch zumal sie in der Ausräumungszone des Inlandeises östlich der Stauchwälle liegen. Und zum anderen ist die Verknüpfung der interglazialen Vorkommen bei Köln (Efferen, Bensberg nach KAISER \& SchüTRUMPF) mit den untersuchten Vorkommen im Raum Krefeld nicht eindeutig.

Möglicherweise zeigt sich über die aufgezählten warmzeitlichen Vorkommen eine Parallele zu der immer noch in Diskussion stehenden Unterteilung der Saale-Eiszeit durch eine zwischengeschaltete Warmzeit (Treene) (Picard 1960; Stremme 1964). Nach Menke (1978) könnte allenfalls noch eine Früh-Drenthe-Warmzeit diskutiert werden. Einen Hinweis zu der Frage, wo am Niederrhein im Liegenden der Niederterrasse weiterhin Eeminterglaziale Bildungen auftreten, geben VAN DER MEENE \& ZAGWIJN (1978). Dieser Frage wird derzeit im Zusammenhang mit der Bearbeitung der Krefelder Mittelterrasse und deren Liegendschichten nachgegangen. Auch im Alpenvorland sind derzeit von FRENZEL (Vortrag in Dijon, Dezember 1978) Fragen aufgegriffen, welche präeemzeitliche Warmzeiten einbeziehen, die jedoch jünger sind als das Holstein-Interglazial.

Die in den folgenden Tabellen 2-4 aufgeführten Taxa sind durchweg mit so geringen Werten aufgetreten, zum Teil als Einzelfunde, daß auf eine Prozentangabe verzichtet werden konnte. 
Tab. 2: Seltene Pollen- und Sporenformen im Profil Stenden

Probe-Nr. Taxa

2

Artemisia

4 Salix, Cupressaceae, Urticaceae, Rubiaceae

5 Cupressaceae, Chenopodiaceae, Rubiaceae

6 cf. Populus, Rubiaceae

$7 \quad$ Cupressaceae, Artemisia, Rubiaceae

8 Cupressaceae, cf. Salix

9 Cupressaceae

10

11

13

15

16

22

23

26

27

29

Frangula alnus, Cupressaceae, Epilobium

Cupressaceae

Cupressaceae, Artemisia, Thalictrum

Succisa, Polygonum spec., Plicapollis pseudoexcelsus

Myrica, Cistaceae, Plicapollis pseudoexcelsus

cf. Carya

cf. Polygalaceae

Cornus

cf. Sciadopitys, Rhamnus frangula, Artemisia, Plicapollis pseudoexcelsus

cf. Salix, Polygonum persicaria-Typ, Ophioglossum vulg.

Tab. 3: Seltene Pollen- und Sporenformen im Profil Eyllsche-Berg

Probe-Nr. Taxa

1a cf. Myrica, Thalictrum, Ostrya-Typ, Polygonum persicaria-Typ

1b Polygonum persicaria-Typ

1c Salix, Cheilanthes, Polypodium viriginianum-T.

2 Cupressaceae

3b Cupressaceae

19 cf. Eucommia

Tab. 4: Seltene Pollen- und Sporenformen im Profil Römerhof II

Probe-Nr. Taxa

$\mathrm{H} 1$ Eucommia

H 2 Salix, Juniperus

$\mathrm{H} 6$ Cupressaceae

H 9 Juniperus, Cupressaceae, Osmunda claytoniana-Typ

H 10 Cupressaceae

H11 Salix, cf. Populus, Ostrya-Typ, Epilobium, Artemisia

H1 14 cf. Myrica, cf. Populus

H 15 Ostrya-Typ, Sambucus

H18 cf. Populus

H19 cf. Populus

H21 cf. Myrica

H 22 Artemisia

Tab. 5: Seltene Pollen- und Sporenformen im Profil Römerhof I

Probe-Nr. Taxa
R 6
R 8
R 9
$\mathrm{R} 14$
$\mathrm{R} 16$
R 17
R 20
R 22
Ostrya-Typ
cf. Populus
Myrica, Artemisia
Myrica
Myrica, cf. Caltha
Ostrya-Typ, Cupressaceae, cf. Myrica, Dipsacaceae, Caltha-Typ
Salix, Cupressaceae, cf. Myrica, cf. Cornus
Cupressaceae 


\section{Schriftenverzeichnis}

Behre, K. E. (1974): Die Vegetation im Spätpleistozän von Osterwanna/Niedersachsen. - Geol. Jb., A 18: 3-48, 8 Abb., 5 Taf.; Hannover.

Bertsch, K. \& SteEger, A. (1927): Jungdiluviale pflanzenführende Ablagerungen am nördlichen Niederrhein. - Sitz.Ber. ü. d. Vers. d. Niederrh. Geol. Ver. f. 1926, 20: 49-65; Bonn.

- \& Steusloff, U. (1931): Fossilführende Schichten in der sogenannten Krefelder Mittelterrasse. - Sitz.Ber. ü. d. Vers. d. Niederrh. Geol. Ver. f. 1929, 23: 1-20; 2 Abb.; Bonn.

Brelie, G. von der, Kilpper, K., \& Teichmüllet R. (1959): Das Pleistozän-Profil von Frimmersdorf an der Erft. - Fortschr. Geol. Rheinld. u. Westf., 4: 179-196; 6 Abb., 2 Tab., 10 Taf.; Krefeld.

BrunNaCKer, K. (1978): Gliederung und Stratigraphie der Quartärterrassen am Niederrhein. Kölner Geogr. Arb., 36: 37-58, 4 Abb., 1 Tab.; Köln.

- Boenigk, W., Dolezalex, B., Kempf, E. K., Koci, A., Meutzen, H., Razi Rad, M. \& WintER, K.-P. (1978): Die Mittelterrassen am Niederrhein zwischen Köln und Mönchengladbach. - Fortschr. Geol. Rheinld. u. Westf., 28: 277-324, 16 Abb., 4 Tab, 1 Taf., Krefeld.

Frenzel, B. (1964): Zur Pollenanalyse von Lössen. - Untersuchungen der Lößprofile von Oberfellabrunn und Stillfried (Niederösterreich). - Eiszeitalter u. Gegenwart, 15: 5-39, 6 Abb., 1 Tab.; OOhringen/Württ.

GrüNER, W. (1975): Stratigraphie der Stauchmoränen und Mittelterrassen bei Krefeld. - Dipl., Arb. Univ. Köln; 175 S., 29 Tab., 2 Beil.; Köln. - [Unveröff.]

KaISER, K. \& SchütrumpF, R. (1960): Zur Gliederung mittel- und jungpleistozäner Schichten in der Niederrheinischen Bucht. - Eiszeitalter u. Gegenwart, 11: 166-185, 5 Abb., 2 Tab.; Ohringen/Württ.

KempF, E. K. (1966): Das Holstein-Interglazial von Tönisberg im Rahmen des niederrheinischen Pleistozäns. - Eiszeitalter u. Gegenwart, 17: 5-60, 7 Abb., 16 Tab.; O'hringen/Württ.

- (1968): Mollusken aus dem Holstein-Interglazial des Niederrheingebietes. - Arch. Moll., 98, 1/2: 1-22, 3 Abb., 4 Taf.; Frankfurt.

KräUsel, R. (1937): Pflanzenreste aus den diluvialen Ablagerungen im Ruhr-Emscher Gebiet. Decheniana, 95, A: 207-240; Bonn.

Lanser, K. (1978): Die „Krefelder Mittelterrasse“ bei Krefeld. - Dipl.-Arb. Köln: 136 S., 2 Abb., 8 Tab., 13 Beilagen; Köln. - [Unveröff.]

van der MeEne, E. A. \& ZaGwiJn, W. H. (1978): Die Rheinläufe im deutsch-niederländischen Grenzgebiet seit der Saale-Kaltzeit. Uberblick neuerer geologischer und pollenanalytischer Untersuchungen. - Fortschr. Geol. Rheinl. u. Westf., 28: 345-359, 4 Abb., 1 Tab.; Krefeld.

Menke, B. (1975): Vegetationsgeschichte und Florenstratigraphie Nordwestdeutschlands im Pliozän und Frühquartär. Mit einem Beitrag zur Biostratigraphie des Weichselfrühglazials. Geol. Jb., A 26: 3-151, 9 Abb., 3 Tab., 8 Taf.; Hannover.

MüLLER, H. (1974): Pollenanalytische Untersuchungen und Jahresschichtenzählung an der holsteinzeitlicher Kieselgur von Munster-Breloh. - Geol. Jb., A 21: 107-140, 10 Abb., 2 Tab.; Hannover.

Steeger, A. (1952): 100 Jahre Eiszeitforschung am Niederrhein. - Der Niederrhein, 19: 5763, 1 Tab.; Krefeld.

Stremme, H.-E. (1964): Die Warmzeiten vor und nach der Warthe-Eiszeit in ihren Bodenbildungen bei Böxlund (westl. Flensburg). - N. Jb. Geol. Paläont. Mh., 4: 237-247, 3 Abb., 2 Tab.; Stuttgart.

Turner, C. (1970): The Middle Pleistocene Deposits at Marks Tey, Essex. - Phil. Trans. Roy. Soc. Lond., B 257: 373-437; London.

URBAN, B. (1978 a): Die Stellung altquartärer Altwasserabsätze in der Niederrheinischen Bucht anhand neuerer paläobotanischer Untersuchungen. - Kölner Geogr. Arb., 36: 69-77, 1 Abb., 1 Tab., 2 Beil.; Köln.

- (1978 b): The Interglacial of Frechen I/Rheinland. - A section of the Tiglian A-type. Geologie en Mijnbouw, 57, (3): 401-406, 4 fig., 1 enclosure; s'Gravenhage. 
- (1978 c): Vegetationsgeschichtliche Untersuchungen zur Gliederung des Altquartärs der Niederrheinischen Bucht. - Sonderveröff. Geol. Inst. Univ. Köln, 34: 165 S., 13 Abb., 5 Tab., 3 Taf., 14 Beil.; Köln.

- (1978 d): Die Stellung des Kärlicher Brockentuffs im Kärlicher Interglazial am Mittelrhein. In: Beiträge zur Quartär- und Landschaftsforschung: 649-654, 1 Abb., 2 Tab.; Wien.

- (1980): Zur Stratigraphie des Frimmersdorf-Interglazials und Krefeld-Interglazials in der Niederrheinischen Bucht. - Decheniana, 133: 224-228, 2 Abb., 1 Tab.; Bonn.

Zagwij, W. H. (1973): Pollenanalytic studies of Holsteinian and Saalian Beds in the Northern Netherlands. - Med. Rijks Geol. Dienst, N.S., 24: 139-156, 13 fig., 2 tab.; enclosures 5-12; Maastricht.

Manuskript eingegangen am 24. 8. 1979 